\title{
ON NUMERICALLY PLURICANONICAL CYCLIC COVERINGS
}

\author{
V. KHARLAMOV AND VIK.S. KULIKOV
}

\begin{abstract}
In this article, we investigate some properties of cyclic coverings $f$ : $Y \rightarrow X$ of complex surfaces of general type $X$ branched along smooth curves $B \subset X$ that are numerically equivalent to a multiple of the canonical class of $X$. The main results concern coverings of surfaces of general type with $p_{g}=0$ and Miyaoka-Yau surfaces; in particular, they provide new examples of multicomponent moduli spaces of surfaces with given Chern numbers as well as new examples of surfaces that are not deformation equivalent to their complex conjugates.
\end{abstract}

\section{INTRODUCTION}

In this article, we investigate some properties of cyclic coverings of algebraic surfaces. Let us recall that there exist two equivalent approaches to define and to treat branched coverings. One is due to Grauert-Remmert-Stein theorem, which insures that the covering is uniquely defined by its unramified part; in particular, if the base of a branched covering is nonsingular, then the branch locus is either empty or a divisor, and each unbranched finite covering of the complement of a divisor extends uniquely to a branched covering with a normal covering variety. The other, also traditional, one, to which we give preference in this paper, is due to the canonical equivalence between finite branched coverings of a given nonsingular (or normal) variety $X$ and the finite field extensions of its rational function field $\mathbb{C}(X)$. A branched covering is called Galois, if the field extension is Galois, and it is called cyclic if the Galois group is cyclic.

Thus, given a finite cyclic cover $Y \rightarrow X$, one can speak on the action of a finite cyclic group $G$ on $Y$ and identify $X$ with the quotient variety $X=Y / G$. It is worth, however, to underline that when we speak on a cyclic Galois group and a cyclic Galois covering, we are not fixing an isomorphism between the Galois group and a group of permutations of a given finite set; in particular, we call two Galois coverings, $f_{1}: Y_{1} \rightarrow X$ and $f_{2}: Y_{2} \rightarrow X$, isomorphic if, and only if, there exists an isomorphism $\phi: Y_{1} \rightarrow Y_{2}$ such that $f_{2} \circ \phi=f_{1}$ and $\phi$ transforms Galois automorphisms in Galois automorphisms.

The cyclic coverings that we consider in this paper are somehow special. Namely, they have a non-empty branch locus $B \subset X$ and we forbid the Galois group action

The first author was partially funded by the ANR-09-BLAN-0039-01 grant of Agence Nationale de la Recherche. The second author was partially supported by grants of NSh-4713.2010.1, RFBR 11-01-00185, and by AG Laboratory HSE, RF government grant, ag. 11.G34.31.0023. 
to have points in $Y$ whose stabilizer is a non-trivial proper subgroup of the Galois group. We call such coverings totally ramified. (Note, that since we consider only the coverings over a smooth $X$, the above restriction forbids also the Galois group to have isolated fixed points in $Y$.)

Note that the assumption to be totally ramified being applied to the points of the ramification divisor $R \subset Y$ implies that $f^{*}(B)=d R, d=\operatorname{deg} f$. Furthermore, a degree $d$ cyclic covering $f: Y \rightarrow X$ is totally ramified (over $X$ ) if, and only if, $f^{*}(B)=d R$ and $f$ is unramified over $X \backslash B$. Note that if $X_{1}$ is birationally equivalent to $X$ then the cyclic covering $f_{1}: Y_{1} \rightarrow X_{1}$, induced by a totally ramified cyclic covering $f: Y \rightarrow X$, is not necessary totally ramified over $X_{1}$ if the branch curve $B \subset X$ of $f$ has singular points.

By a numerically multi-canonical cyclic covering we understand a totally ramified cyclic covering whose branch curve is non-singular and numerically equivalent to a multiple of the canonical class.

The main results of the article can be divided into three groups.

First, we consider the moduli space of surfaces with given square of the canonical class, $K_{Y}^{2}$, and given arithmetic genus, $p_{a}(Y)$, and, under assumption that it contains surfaces given by $d$-sheeted totaly ramified numerically multi-canonical cyclic coverings $f: Y \rightarrow X$ of a surface of general type $X$, provide a lower bound for the number of connected components of such a moduli space depending on the number of elements of the torsion group Tor $H^{2}(X, \mathbb{Z})$ (see Theorem 1, Proposition 3, Remark 4, and Corollaries 3, 41).

Second, we investigate the degree of the canonical map $\varphi_{K_{Y}}: Y \rightarrow \mathbb{P}^{p_{g}(Y)-1}$ for surfaces $Y$ given as two-sheeted totaly ramified numerically multi-canonical cyclic coverings $f: Y \rightarrow X$, where $X$ is a surface of general type with $p_{g}(X)=0$ (see Theorem 3, Corollary 1, and Propositions 4- 6).

Third, we show that if a Miyaoka-Yau surface $X$ (that is, a surface of general type with $c_{1}^{2}=3 c_{2}$ ) has no anti-holomorphic automorphisms (such a property is shared, for example, by all fake projective planes, see [9]) and if a surface $Z$ is deformation equivalent to $Y$ given as a totaly ramified numerically multi-canonical cyclic covering $f: Y \rightarrow X$, then $Z$ also has no anti-holomorphic automorphisms (see Theorem 7). Note that, together with the known examples (see, [12], [9], [10], [6]) of pairs of complex surfaces $\left(Z, Z^{\prime}\right)$ that are not deformation equivalent despite being diffeomoprhic (with preserving the orientation), the surfaces $Y$ involved in Theorem 7 and their complex conjugates, $\bar{Y}$, give infinite series of new examples of diffeomoprhic, but not deformation equivalent, surfaces. Besides, we prove that those connected components $M$ of the moduli space of surfaces that contain surfaces $Y$, obtained as two-sheeted totaly ramified numerically multi-canonical cyclic coverings $f: Y \rightarrow X$ of a Miyaoka-Yau surface $X$ branched along a curve $B$ numerically equivalent to $2 m K_{X}$, are irreducible varieties of complex dimension $\operatorname{dim} M=m(2 m-1) K_{X}^{2}+p_{g}(X)$ and Kodaira dimension $\kappa(M)=-\infty$, moreover, if the irregularity $q(X)=0$ then $M$ is an unirational variety (see Theorem [6 and also Remark 7). 
Up to ou knowledge, there are a very few, referred to in the literature, examples of complex surfaces of general type with such a nontrivial action of a group that is deforming simultaneously with any deformation of complex structure (cf., [10]). As a consequence of the proof of Theorem [6 we provide infinite series of such surfaces with action of the group $\mathbb{Z} / 2 \mathbb{Z}$ (see Remark 7 ).

\section{Totally RAMified CYCliC COVERINGS: FUndAMENTAL GROUPS AND CLASSIFICATION}

Recall that each continuous map $f: V \rightarrow W$ of path connected topological spaces defines the homomorphism of fundamental groups, $f_{*}: \pi_{1}(V, q) \rightarrow \pi_{1}(W, p)$, for any pair of points, $q \in V$ and $p \in W$ with $p=f(q)$, and that for any pair of basic points, $\left(q^{\prime}, p^{\prime}\right)$ and $\left(q^{\prime \prime}, p^{\prime \prime}\right)$ connected by the pathes, $h$ in $V$ and $f(h)$ in $W$, these homomorphisms are conjugated by change-of-basepoint homomoprhisms $\beta_{h}$ and $\beta_{f(h)}$ in the sense that $f_{*}^{\prime \prime} \circ \beta_{h}=\beta_{f(h)} \circ f_{*}^{\prime}$. Note that all these homomorphisms $f_{*}$ induce one and the same homomorphism

$$
H_{1}(V, \mathbb{Z}) \simeq \pi_{1}(V, q) /\left[\pi_{1}(V, q), \pi_{1}(V, q)\right] \rightarrow H_{1}(W, \mathbb{Z}) \simeq \pi_{1}(W, p) /\left[\pi_{1}(W, p), \pi_{1}(W, p)\right]
$$

at the level of the first homology groups. Below, when the choice of the base points $p \in W$ and $q \in f^{-1}(p)$ in the fundamental groups $\pi_{1}(V, q)$ and $\pi_{1}(W, p)$ is not essential for the proof, we omit mentioning the base points and denote the fundamental groups by $\pi_{1}(V)$ and $\pi_{1}(W)$, respectively.

Proposition 1. Let $X$ be an irreducible smooth projective surface, let $B \subset X$ be an irreducible reduced smooth curve divisible by $d$ as an element of Pic $(X)$, and let $f$ : $Y \rightarrow X$ be a d-sheeted totally ramified cyclic covering branched along $B$. If $\left(B^{2}\right)_{X}>0$ then the homomorphism $f_{*}: \pi_{1}(Y) \rightarrow \pi_{1}(X)$ induced by $f$ is an isomorphism.

Lemma 1. Let $B$ be an irreducible reduced smooth curve on an irreducible smooth projective surface $X$. If $\left(B^{2}\right)_{X}>0$, then the kernel $K$ of the epimorphism $\pi_{1}(X \backslash B) \rightarrow$ $\pi_{1}(X)$ is a cyclic group and it is a subgroup of the center of $\pi_{1}(X \backslash B)$.

Proof. Let $N \subset X$ be the closure of a tubular neighbourhood of $B$. Then $N$ has a structure of a locally trivial $C^{\infty}$-fibration over $B$ with fiber $D=\{z \in \mathbb{C}|| z \mid \leqslant 1\}$. If we delete one fiber of this fibration (say, over a point $b \in B$ ), then we get a trivial fibration $N_{0} \simeq D \times B_{0}$ over $B_{0}=B \backslash\{b\}$. Consider the section $B_{1}=B_{0} \times\{z=1\}$ of the latter fibration and choose a point $p \in B_{1}$.

Let $\gamma \in G=\pi_{1}(X \backslash B, p)$ be an element represented by the loop $\partial D$, where $\partial D$ is the boundary of the fibre of $N \rightarrow B$ containing $p$. We have the exact sequence of groups

$$
1 \rightarrow K \rightarrow G \rightarrow \pi_{1}(X, p) \rightarrow 1,
$$

where $K$ is the normal closure of $\gamma$ in $G$.

By Nori's Weak Lefschetz Theorem (see [18], Proposition 3.27), $K$ is a finitely generated abelian group and its centralizer $C(K)$ in $G$ is a subgroup of finite index. 
On the other hand, we have $\pi_{1}\left(N_{0} \backslash B_{0}, p\right)=\pi_{1}\left(B_{1}, p\right) \times\left\{\gamma^{n} \mid n \in \mathbb{Z}\right\}$. Again by Nori's Weak Lefschetz Theorem (see [18], Proposition 3.26), the embedding $i$ : $B \hookrightarrow X$ induces an epimorphism $i_{*}: \pi_{1}(B) \rightarrow \pi_{1}(X)$. Therefore, the elements of $\pi_{1}\left(B_{1}, p\right)$ generate the group $\pi_{1}(X, p)$ and the embedding $N_{0} \backslash B_{0} \hookrightarrow X \backslash B$ induces an epimorphism

$$
\pi_{1}\left(N_{0} \backslash B_{0}, p\right)=\pi_{1}\left(B_{1}, p\right) \times\left\{\gamma^{n} \mid n \in \mathbb{Z}\right\} \rightarrow \pi_{1}(X \backslash B, p) .
$$

Hence, the normal closure $K$ of $\gamma$ in $\pi_{1}(X \backslash B, p)$ is nothing but the cyclic group generated by $\gamma$ and, furthermore, $K$ is contained in the center of $\pi_{1}(X \backslash B, p)$.

Proof of Proposition 1. The covering $f: Y \rightarrow X$ induces an embedding of the fundamental groups $f_{*}: \pi_{1}\left(Y \backslash f^{-1}(B)\right) \hookrightarrow \pi_{1}(X \backslash B)$ such that $\bar{G}=f_{*}\left(\pi_{1}\left(Y \backslash f^{-1}(B)\right)\right)$ is a subgroup of $G=\pi_{1}(X \backslash B)$ of index $d=\operatorname{deg} f$.

Let $\gamma$ denote, as in the proof of Lemma 1, a generator of $K$. Since $f^{*}(B)=d R$, we have $\gamma^{i} \notin \bar{G}$ for $1 \leqslant i \leqslant d-1$ and $\gamma^{d} \in \bar{G}$. Therefore, $G=\bar{G} \cup \gamma \bar{G} \cup \cdots \cup \gamma^{d-1} \bar{G}$. On the other hand, $\pi_{1}(Y) \simeq \bar{G} /\left\langle\gamma^{d}\right\rangle$. Exact sequence (1) gives rise to the exact sequence

$$
1 \rightarrow K /\left\langle\gamma^{d}\right\rangle \rightarrow G /\left\langle\gamma^{d}\right\rangle \rightarrow \pi_{1}(X) \rightarrow 1
$$

The group $\bar{G} /\left\langle\gamma^{d}\right\rangle \simeq \pi_{1}(Y)$ naturally imbeds into $G /\left\langle\gamma^{d}\right\rangle$, so that Proposition 1 follows now from exact sequence (2) and the equality

$$
G /\left\langle\gamma^{d}\right\rangle=\pi_{1}(Y) \cup \gamma \pi_{1}(Y) \cup \cdots \cup \gamma^{d-1} \pi_{1}(Y) .
$$

Let $C_{1}$ and $C_{2}$ be two divisors on a surface $X$. We will use the notation $C_{1} \sim C_{2}$ if $C_{1}$ and $C_{2}$ are linear equivalent and $C_{1} \equiv C_{2}$ if $C_{1}$ and $C_{2}$ are numerically equivalent. Denote by $\operatorname{Tor}_{d} \operatorname{Pic}(X)$ the subgroup of the Picard group $\operatorname{Pic}(X)$ consisting of the elements whose order is a divisor of $d$, and by $N_{X, d}=\left|\operatorname{Tor}_{d} \operatorname{Pic}(X)\right|$ the order of $\operatorname{Tor}_{d} \operatorname{Pic}(X)$. Given a divisor $B$ whose divisor class is divisible by $d \in \mathbb{N}$, we denote by $(B)_{d} \subset \operatorname{Pic}(X)$ the set of divisor classes $\beta$ such that $B \sim d \beta$. Clearly, $(B)_{d}$ is a principal homogeneous space over $\operatorname{Tor}_{d} \operatorname{Pic}(X)$.

The following lemma is well-known. Since we did not find an appropriate reference, we supply this lemma with a proof.

Lemma 2. Let $B \subset X$ be an irreducible reduced curve. If $B$, as an element of $\operatorname{Pic}(X)$, is divisible by $d$ (that is $B \sim d C$ for some divisor $C$ ), then there is a natural bijection between $(B)_{d}$ and the set of isomorphism classes of d-sheeted totally ramified cyclic coverings $f_{i}: Y_{i} \rightarrow X$ branched along $B$. If $\left[C_{i}\right] \in(B)_{d}$ is the divisor class corresponding to $f_{i}$ under this bijection, then $\left[R_{i}\right]=f_{i}^{*}\left[C_{i}\right]$ and $d\left[C_{i}\right]=[B]$.

If $B$ is a smooth curve, then each of these $Y_{i}$ is a smooth surface. 
Proof. By definition, a finite morphism $f: Y \rightarrow X$ is a branched $d$-sheeted cyclic covering of a smooth surface $X$ if $Y$ is a normal surface and $f^{*}: \mathbb{C}(X) \hookrightarrow \mathbb{C}(Y)$ is a finite Galois extension of fields with Galois group $\operatorname{Gal}(Y / X) \simeq \mathbb{Z} / d \mathbb{Z}$. Let $h^{*}$ be a generator of $\operatorname{Gal}(Y / X)$. In accordance with so called Hilbert's theorem 90, in the field $\mathbb{C}(Y)$, considered as the vector space over $\mathbb{C}(X)$, one can choose a basis $w_{0}=1, w_{1}, \ldots, w_{d-1}$ over $\mathbb{C}(X)$ such that $h^{*}\left(w_{i}\right)=\mu^{i} w_{i}$, where $\mu$ is a primitive $d$ root of unity. Hence, we can put $w=w_{1}$ and conclude that each branched $d$-sheeted cyclic covering can be seen as an extension $\mathbb{C}(Y)=\mathbb{C}(X)(w)$ with $h^{*}(w)=\mu w$, and $w^{d}=g \in \mathbb{C}(X)$ for some function $g$. The reverse statement is also straightforward.

The automorphism $h^{*}$ defines an automorphism $h: Y \rightarrow Y$, while the branch locus of $f$ consists of those irreducible curves $D_{i}$ that participate in the principal divisor $(g)=\sum_{i=0}^{n} a_{i} D_{i} \in \operatorname{Div}(X), a_{i} \in \mathbb{Z}$, with coefficients $a_{i} \not \equiv 0 \bmod d$. Furthermore, $\operatorname{gcd}\left(a_{i}, d\right)$ is equal to the number of points in the inverse image of a generic point in $D_{i}$. Therefore, in our case there is a unique curve $D_{i}$ (say, $D_{0}$; and it must be the branch curve $B$ ) for which $a_{0} \not \equiv 0 \bmod d$ and, moreover, $a_{0}$ and $d$ are coprime. Thus, we can find an integer $b$ coprime with $d$ such that $\left(g^{b}\right)=B-d C$ for some divisor $C \in \operatorname{Div}(X)$. Therefore, choosing a function $\tilde{g} \in \mathbb{C}(X)$ and replacing $w$ by $w^{b} \tilde{g}, g$ by $\tilde{g}^{d} g^{b}$, and $\mu$ by $\mu^{b}$, we get a presentation $\mathbb{C}(Y)=\mathbb{C}(X)(w), h^{*}(w)=\mu w, w^{d}=g$ with $(g)=B-d C^{\prime}$, where $C^{\prime}$ is any given divisor linear equivalent to $C$. This provides a natural bijection between $(B)_{d}$ and the set of isomorphism classes of $d$-sheeted cyclic coverings of $X$ branched along $B$.

If $R$ is the ramification locus of $f, R=f^{-1}(B)$ and $f^{*}(B)=d R$ for an irreducible curve $B$, then $\left(w^{d}\right)=\left(f^{*}(g)\right)=d R-d f^{*}(C)$. Therefore, $(w)=R-f^{*}(C)$.

Remark 1. To enumerate the branched coverings as in Lemma 2, it is often convenient to choose (as in the proof of Lemma 2) as "a base point" some divisor $C$ such that $B \sim d C$ and then make use of the bijection that associates with $\alpha_{i} \in \operatorname{Tor}_{d} \operatorname{Pic} X$ the $d$-sheeted cyclic coverings defined by $w^{d}=g_{i}$, where $\left(g_{i}\right)=B-d C_{i}$ with $C_{i} \sim C+\alpha_{i}$.

Remark 2. Lemma 2 and Remark 1 hold also if $B$ is not necessary irreducible, but reduced, curve and $d=2$.

Let $f: Y \rightarrow X$ be a $d$-sheeted totally ramified cyclic covering $f: Y \rightarrow X$ branched along a smooth irreducible curve $B \subset X$ with $\left(B^{2}\right)_{X}>0$. By Proposition 1, the map $f$ induces an isomorphism between the fundamental groups, $\pi_{1}(Y)$ and $\pi_{1}(X)$. Therefore, it induces also an isomorphism $f_{*}$ between $H_{1}(Y, \mathbb{Z})$ and $H_{1}(X, \mathbb{Z})$ and, in particular, between their torsion subgroups, Tor $H_{1}(Y, \mathbb{Z})$ and $\operatorname{Tor} H_{1}(X, \mathbb{Z})$, which in its turn implies, by the universal coefficient theorem (that gives $\operatorname{Tor} H_{1}(V, \mathbb{Z})=$ $\operatorname{Ext}\left(H_{1}(V ; \mathbb{Z}), \mathbb{Z}\right)=$ Tor $H^{2}(V, \mathbb{Z})$ for every compact manifold $\left.V\right)$ and its functoriality, that $f^{*}: \operatorname{Tor} H^{2}(X, \mathbb{Z}) \rightarrow \operatorname{Tor} H^{2}(Y, \mathbb{Z})$ is an isomorphism as well.

In fact, even a stronger statement holds under our assumptions. Indeed, the exponential exact sequence of sheaves

$$
0 \rightarrow \mathbb{Z} \rightarrow \mathcal{O}_{Y} \rightarrow \mathcal{O}_{Y}^{*} \rightarrow 0
$$


provides a short exact sequence

$$
0 \rightarrow \operatorname{TorPic}^{0}(Y) \rightarrow \operatorname{Tor} \operatorname{Pic}(Y) \stackrel{\delta}{\rightarrow} \operatorname{Tor} H^{2}(Y, \mathbb{Z}) \rightarrow 0,
$$

where $\operatorname{Pic}^{0}(Y)$ is the connected component of 0 in $\operatorname{Pic}(Y)$ and $\delta$ is the connecting (first Chern class) homomorphism. Hence, using the functorial isomorphism $\operatorname{Pic}^{0}(V)=$ $H^{0,1}(V) /\left(H^{1}(V ; \mathbb{Z})^{0,1}\right.$ and the five lemma applied to the diagram formed by the above short exact sequence and its copy written for $X$, one proves that $f^{*}$ : Tor Pic $(X) \rightarrow$ Tor $\operatorname{Pic}(Y)$ is also an isomorphism.

In what follows, we use only $\operatorname{Tor} H^{2}(V, \mathbb{Z})$ and, for shortness, abbreviate this notation to $\operatorname{Tor}(V)$.

\section{NumeriCALly MULTI-CANONICAL CYCLIC COVERINGS}

In this section we fix an integer $d \geqslant 2$ and a smooth irreducible curve $B \equiv d m K_{X}$ on a nonsingular surface $X$. A $d$-sheeted totally ramified cyclic covering $f: Y \rightarrow$ $X$ branched along $B$ and defined by (see Remark 1) a divisor class $C+\alpha, \alpha \in$ $\operatorname{Tor}_{d} \operatorname{Pic}(X)$, is called $(d, m)$-canonical if $C \sim m K_{X}$ and $d \alpha=0$, pure $(d, m)$-canonical if $C \sim m K_{X}$ and $\alpha=0$, and numerically $(d, m)$-canonical if $C \equiv m K_{X}$. Note that if $f$ is $(d, m)$-canonical, then $B \in\left|d m K_{X}\right|$.

In the following, given a divisor $D$ on a surface $X$ we denote by $\varphi_{D}: X \rightarrow \mathbb{P}^{\operatorname{dim}|D|}$ the rational map defined by the complete linear system $|D|$.

The famous Bombieri Theorem ([3]) states that if $m \geqslant 5$ then for a smooth minimal (that is, without $(-1)$-curves) projective surface $X$ the $m$-canonical map $\varphi_{m K_{X}}: X \rightarrow$ $\mathbb{P}^{P_{m}-1}$ is a birational morphism onto its image, where $P_{m}=\operatorname{dim} H^{0}\left(X, \mathcal{O}_{X}\left(m K_{X}\right)\right)$. If to examine the proof of this theorem, one can see that except Ramanujam vanishing theorem only the numerical properties of the canonical class $K_{X}$ are used in the proof. Therefore Bombiery Theorem is true not only for $m$-canonical maps, but also for maps $\varphi_{D_{m}}: X \rightarrow \mathbb{P}^{P_{m}-1}$, where $D_{m}$ is a divisor numerically equivalent to $m K_{X}$. In particular, if $d m \geqslant 5$ and $D \equiv d m K_{X}$, then by Bertini Theorem, a generic curve $B \in|D|$ is non-singular and irreducible.

Proposition 2. Let $f: Y \rightarrow X$ be a numerically $(d, m)$-canonical totally ramified cyclic covering of a surface $X$. Then $Y$ has the following invariants:

$$
\begin{gathered}
p_{a}(Y)=d p_{a}(X)+\frac{d(d-1) m((2 d-1) m+3)}{12} K_{X}^{2}, \\
K_{Y}^{2}=d(d m-m+1)^{2} K_{X}^{2},
\end{gathered}
$$

and $q(Y)=q(X)$, where $p_{a}=p_{g}-q+1$ is the arithmetic genus of a surface. 
Proof. We have $d K_{Y} \sim f^{*}\left(d K_{X}+(d-1) B\right) \equiv f^{*}\left(d(d m-m+1) K_{X}\right)$. Therefore $K_{Y}^{2}=d(d m-m+1)^{2} K_{X}^{2}$.

It follows from Proposition 1 that $q(Y)=q(X)$.

Let $e(V)$ denote the topological Euler characteristic of a variety $V$. We have

$$
K_{X}^{2}+e(X)=12 p_{a}(X)
$$

by Noether formula and, by adjunction formula,

$$
-e(B)=2 g(B)-2=\left(B, B+K_{X}\right)_{X}=\left(d m K_{X},(d m+1) K_{X}\right)_{X}=d m(d m+1) K_{X}^{2} .
$$

Since $f^{*}(B)=d R$, we have

$$
\begin{aligned}
e(Y)= & d(e(X)-e(B))+e(B)= \\
& d\left(12 p_{a}(X)-K_{X}^{2}+d m(d m+1) K_{X}^{2}\right)-d m(d m+1) K_{X}^{2}= \\
& 12 d p_{a}(X)+d[(d-1)(d m+1) m-1] K_{X}^{2} .
\end{aligned}
$$

Hence,

$$
\begin{aligned}
e(Y)+K_{Y}^{2}= & 12 d p_{a}(X)+d[(d-1)(d m+1) m-1] K_{X}^{2}+d(d m-m+1)^{2} K_{X}^{2}= \\
& 12 d p_{a}(X)+d\left[(d-1)(d m+1) m-1+(d m-m+1)^{2}\right] K_{X}^{2}= \\
& 12 d p_{a}(X)+d(d-1) m[(2 d-1) m+3] K_{X}^{2}
\end{aligned}
$$

and therefore

$$
p_{a}(Y)=d p_{a}(X)+\frac{d(d-1) m[(2 d-1) m+3]}{12} K_{X}^{2}
$$

By Proposition 2, the invariants $K_{Y}^{2}$ and $p_{a}(Y)$ are the same for all numerically $(d, m)$-canonical totally ramified cyclic coverings $f: Y \rightarrow X$ with fixed $d$ and $m$. We denote them by $k_{X, d, m}$ and $p_{X, d, m}$, respectively. The moduli space of surfaces $Z$ with given invariants $K_{Z}^{2}=k$ and $p_{a}(Z)=p$ will be denoted by $\mathcal{M}_{k, p}$.

Theorem 1. Let $X$ be a surface of general type. If there is an element $\alpha \in \operatorname{Tor}_{d} \operatorname{Pic}(X)$ such that $\delta(\alpha)$ is not divisible by $d$ in Tor $(X)$, then for each integer $n \geqslant 1$ the moduli space $\mathcal{M}_{k, p}$ with $k=k_{X, d, d n+1}$ and $p=p_{X, d, d n+1}$ consists of at least two connected components.

Proof. Let us choose a divisor $C \sim(d n+1) K_{X}$. Consider two $(d, d n+1)$-canonical totally ramified cyclic coverings $f_{i}: Y_{i} \rightarrow X, i=1,2$, branched along a smooth irreducible curve $B \in\left|d(d n+1) K_{X}\right|$, where $f_{1}$ is the pure $(d, d n+1)$-canonical and $f_{2}$ is the $(d, d n+1)$-canonical totally ramified cyclic covering defined by $C+\alpha$ where $\alpha$ belongs to $\operatorname{Tor}_{d} \operatorname{Pic}(X)$ and $\delta(\alpha)$ is not divisible by $d$ in $\operatorname{Tor}(X)$. For $n \in \mathbb{N}$ the existence of such a curve $B$ follows from the inequality $d(d n+1) \geqslant 6$.

Consider the covering $f_{1}$. It is given by adding to the field $\mathbb{C}(X)$ a function $w_{1}$ such that $w_{1}^{d}=g_{1}$ and $\left(g_{1}\right)=B-d C$. Since

$$
R_{1} \sim f_{1}^{*}(C) \sim f^{*}\left((d n+1) K_{X}\right),
$$


then from the projection formula for the canonical divisors we get $K_{Y_{1}} \sim f_{1}^{*}\left(K_{X}\right)+(d-1) R_{1} \sim f_{1}^{*}\left(K_{X}\right)+(d-1) f^{*}\left((d n+1) K_{X}\right) \sim d(d n-n+1) f^{*}\left(K_{X}\right)$. In particular, $K_{Y_{1}}$ is divisible by $d$ in $\operatorname{Pic}\left(Y_{1}\right)$, and hence its cohomology class is divisible by $d$ in $H^{2}\left(Y_{1}, \mathbb{Z}\right)$.

The covering $f_{2}$ is given by adding to the field $\mathbb{C}(X)$ a function $w_{2}$ such that $w_{2}^{d}=g_{2}$ and $\left(g_{2}\right)=B-d C_{2}$ with $C_{2} \sim(d n+1) K_{X}+\alpha$. Due to Lemma 2, $R_{2} \sim f_{2}^{*}\left(C_{2}\right) \sim f_{2}^{*}\left((d n+1) K_{X}+\alpha\right)$. Hence,

$$
\begin{aligned}
K_{Y_{2}} \sim & f_{2}^{*}\left(K_{X}\right)+(d-1) R_{2} \sim f_{2}^{*}\left(K_{X}\right)+(d-1) f_{2}^{*}\left((d n+1) K_{X}+\alpha\right) \sim \\
& d(d n-n+1) f_{2}^{*}\left(K_{X}\right)+(d-1) f_{2}^{*}(\alpha) .
\end{aligned}
$$

Since $f_{2}^{*}: \operatorname{Tor}(X) \rightarrow \operatorname{Tor}\left(Y_{2}\right)$ is an isomorphism and $\delta(\alpha) \in \operatorname{Tor}(X)$ is not divisible by $d$, this shows that the cohomology class of $K_{Y_{2}}$ is not divisible by $d$ in $H^{2}\left(Y_{2}, \mathbb{Z}\right)$.

In view of different divisibility of the cohomology classes of their canonical divisors, $Y_{1}$ and $Y_{2}$ can not belong to the same connected component in the moduli space.

Remark 3. Theorem 1 is true also in the case $n=0$ if there is a smooth irreducible curve $B \in\left|d K_{X}\right|$. From Theorem 5.2 in [1], Proposition 3 in [19], and Bertini Theorem, it follows that a generic curve $B \in\left|d K_{X}\right|$ is smooth and irreducible if $d \geqslant 5$, or if $d=4$ and $K_{X}^{2} \geqslant 2$, or if $d=3$ and $K_{X}^{2} \geqslant 3$, or if $d=2$ and $K_{X}^{2} \geq 5$.

Remark 4. Note that if $X_{1}$ and $X_{2}$ are two surfaces of general type such that $K_{X_{1}}^{2}=K_{X_{2}}^{2}$ and $p_{a}\left(X_{1}\right)=p_{a}\left(X_{2}\right)$, but $\pi_{1}\left(X_{1}\right)$ is not isomorphic to $\pi_{1}\left(X_{2}\right)$, and if $f_{1}: Y_{1} \rightarrow X_{1}$ and $f_{2}: Y_{2} \rightarrow X_{2}$ are numerically $(d, m)$-canonical totally ramified cyclic coverings, then, by Proposition 1, $Y_{1}$ and $Y_{2}$ belong to different connected components of the moduli space $\mathcal{M}_{k, p}$ with $k=k_{X_{1}, d, m}$ and $p=p_{X_{1}, d, m}$.

Let $X$ be a Campedelli surface, that is, $X$ is a surface of general type with $p_{g}=0$, $K_{X}^{2}=2$, and $\pi_{1}(X) \simeq(\mathbb{Z} / 2 \mathbb{Z})^{3}$. A totally ramified numerically $(2,1)$-canonical cyclic covering $f: Y \rightarrow X$ is a $(2,1)$-canonical covering since each non-zero element of $\operatorname{Tor}(X) \simeq(\mathbb{Z} / 2 \mathbb{Z})^{3}$ is not divisible by two. By Proposition 2 , a totally ramified $(2,1)$ canonical cyclic covering $Y$ of $X$ has the following invariants: $K_{Y}^{2}=16$ and $p_{a}=4$. Note also that, by Lemma 2, for given non-singular curve $B \in\left|2 K_{X}\right|$ there exist eight different totally ramified $(2,1)$-canonical cyclic coverings of $X$ branched along $B$.

Proposition 3. There are exactly two connected components of the moduli space $\mathcal{M}_{16,4}$ to which belong the totally ramified $(2,1)$-canonical cyclic coverings of Campedelli surfaces.

Proof. Recall that every Campedelli surface $X$ can be obtained as follows (see, for example, [8]). Let $\widetilde{L}=L_{1} \cup \cdots \cup L_{7}$ be a line arrangement in $\mathbb{P}^{2}$ consisting of seven lines. We numerate them by the non-zero elements $\alpha_{i}=\left(a_{i, 1}, a_{i, 2}, a_{i, 3}\right) \in(\mathbb{Z} / 2 \mathbb{Z})^{3}$ and make two assumptions: the arrangement $\widetilde{L}$ has no $r$-fold points with $r \geqslant 4$; and if $\widetilde{L}$ has a tripe point $p_{\alpha_{i_{1}}, \alpha_{i_{2}}, \alpha_{i_{3}}}=L_{\alpha_{i_{1}}} \cap L_{\alpha_{i_{2}}} \cap L_{\alpha_{i_{3}}}$, then $\alpha_{i_{1}}+\alpha_{i_{2}}+\alpha_{i_{3}} \neq 0$. Consider the Galois covering $\widetilde{g}: \widetilde{X}^{3} \rightarrow \mathbb{P}^{2}$ with Galois group $\operatorname{Gal}\left(\tilde{X} / \mathbb{P}^{2}\right) \simeq(\mathbb{Z} / 2 \mathbb{Z})^{3}$, that is, 
branched in $\widetilde{L}$ and defined by the epimorphism $\varphi: H_{1}\left(\mathbb{P}^{2} \backslash \widetilde{L}, \mathbb{Z}\right) \rightarrow G=(\mathbb{Z} / 2 \mathbb{Z})^{3}$ given by $\varphi\left(\lambda_{i}\right)=\alpha_{i}$, where $\lambda_{i}$ is the element of $H_{1}\left(\mathbb{P}^{2} \backslash \widetilde{L}, \mathbb{Z}\right)$ represented by a small circle around $L_{\alpha_{i}}$.

The only singular points of $\widetilde{X}$ are the points lying over the triple points $p_{\alpha_{i_{1}}, \alpha_{i_{2}}, \alpha_{i_{3}}}$, and $X$ that is the resolution of singularities of $\widetilde{X}$ is a Campedelli surface. To resolve the singularities of $\widetilde{X}$, it suffices to blow up the triple points of $\widetilde{L}$. The composition $\sigma: \widetilde{\mathbb{P}}^{2} \rightarrow \mathbb{P}^{2}$ of blowups with centers at the triple points of $\widetilde{L}$ induces the Galois covering $g: X \rightarrow \widetilde{\mathbb{P}}^{2}$ and we have ([8]) $2 K_{X} \sim g^{*}(L)$, where $L$ is a line in $\mathbb{P}^{2}$. (If $\widetilde{L}$ has no triple points, then $g=\widetilde{g}$.)

Let $X$ be defined by a Campedelli arrangement $\widetilde{L}$ and let $f$ be branched along $B=g^{-1}\left(L_{8}\right)$, where a line $L_{8} \not \subset \widetilde{L}$. Without loss of generality, we can assume that the line arrangement $\mathcal{L}=\widetilde{L} \cup L_{8}$ is generic. Then the fundamental group $\pi_{1}\left(\mathbb{P}^{2} \backslash \mathcal{L}\right)$ is abelian and hence $h=g \circ f: Y \rightarrow \mathbb{P}^{2}$ is a $(\mathbb{Z} / 2 \mathbb{Z})^{4}$-Galois covering of $\mathbb{P}^{2}$ branched along $\mathcal{L}$ and defined by an epimorphism $\psi: H_{1}\left(\mathbb{P}^{2} \backslash \mathcal{L}, \mathbb{Z}\right) \rightarrow(\mathbb{Z} / 2 \mathbb{Z})^{4}$ given by $\psi\left(\lambda_{i}\right)=\widetilde{\alpha}_{i}=\left(a_{i, 1}, a_{i, 2}, a_{i, 3}, a_{i, 4}\right)$ for some $a_{i, 4} \in \mathbb{Z} / 2 \mathbb{Z}, i=1, \ldots, 7$, and $\psi\left(\lambda_{8}\right)=\widetilde{\alpha}_{8}=(0,0,0,1)$, where $\lambda_{8}$ is the element represented by a small circle around $L_{8}$. Note that $\psi$ is defined by $h$ up to automorphisms of $(\mathbb{Z} / 2 \mathbb{Z})^{4}$ (that is, by the choice of the basis in $\left.(\mathbb{Z} / 2 \mathbb{Z})^{4}\right)$. We have $\sum_{i=1}^{8} \lambda_{i}=0$ in $H_{1}\left(\mathbb{P}^{2} \backslash \mathcal{L}, \mathbb{Z}\right)$. Therefore the subset $M=\left\{\widetilde{\alpha}_{i} \subset \mathbb{P}_{\mathbb{Z} / 2 \mathbb{Z}}^{3} \mid i=1, \ldots, 8\right\}$ of the projective space $\mathbb{P}^{3}=\mathbb{P}_{\mathbb{Z} / 2 \mathbb{Z}}^{3}$ over the field $\mathbb{Z} / 2 \mathbb{Z}$ is totally even, that is, it satisfies the following property: for each plane $\mathbb{P}^{2} \subset \mathbb{P}^{3}$ the intersection $M \cap \mathbb{P}^{2}$ consists of even number of points.

Lemma 3. Up to the action of $P G L(4, \mathbb{Z} / 2 \mathbb{Z})$, there exist exactly two different totally even subsets $M$ of the projective space $\mathbb{P}^{3}$ over the field $\mathbb{Z} / 2 \mathbb{Z}$ consisting of eight points, namely, either $M=\mathbb{A}^{3}$ (type $I$ ), or

$$
\begin{aligned}
M=\{ & (1,0,0,0),(1,1,0,0),(0,1,0,0),(1,0,1,0),(0,0,1,0), \\
& (0,1,1,0),(1,1,1,1),(0,0,0,1)\}
\end{aligned}
$$

(type $I I)$.

Proof. Type $I$ corresponds to the case when there is a plane $P \subset \mathbb{P}^{3}$ (say, $P$ is given by equation $a_{4}=0$ ) such that $M \cap P=\emptyset$ and in this case

$$
M=\mathbb{A}^{3}=\left\{\widetilde{\alpha}=\left(a_{1}, a_{2}, a_{3}, a_{4}\right) \in \mathbb{P}^{3} \mid a_{4}=1\right\} .
$$

Assume that there is not a plane $P$ such that $M \cap P=\emptyset$. For each plane $P_{i} \subset \mathbb{P}^{3}$ denote by $n_{i}=\left|M \cap P_{i}\right|$ the number of points of the intersection $M \cap P_{i}$. Each number $n_{i}$ is even, $0<n_{i} \leqslant 6$.

First of all, let us show that the condition $n_{i}=6$ for some $i$ (say, $n_{1}=6$ ) is equivalent to the condition that there exists a line $l \subset M$ (and in this case $|M \cap l|=3$ ). Indeed, if $n_{1}=6$ then there is a unique point $\widetilde{\alpha}_{0} \in P_{1}$ such that $\widetilde{\alpha}_{0} \notin M$, since $\left|P_{1}\right|=7$. Therefore for any line $l \subset P_{1}$ not passing through $\widetilde{\alpha}_{0}$ we have $l \subset M$. Conversely, for a line $l \subset M$ consider the pencil of planes passing through $l$. It 
consists of three planes, say, $P_{1}, P_{2}$, and $P_{3}$. We have $n_{i} \geqslant 4$ for $i=1,2,3$ and $\left(n_{1}-3\right)+\left(n_{2}-3\right)+\left(n_{3}-3\right)=8-3=5$, that is, $n_{1}+n_{2}+n_{3}=14$. Up to permutation, this equation has the only one solution consisting of even numbers and satisfying inequalities $n_{i} \geqslant 4$, namely, $n_{1}=6$ and $n_{2}=n_{3}=4$.

Let us show that $M$ is of type $I I$ if there is a plane $P$ such that $|M \cap P|=6$. Without loss of generality, we can assume that $P$ is given by equation $a_{4}=0$ and the points $\widetilde{\alpha}_{7}$ and $\widetilde{\alpha}_{8}$ lying in $M \backslash P$ have coordinates: $\widetilde{\alpha}_{7}=(1,1,1,1)$ and $\widetilde{\alpha}_{8}=(0,0,0,1)$. Since $|M \cap P|=6$ and $|P|=7$, to prove that $M$ is of type $I I$, it suffices to show that the point $\widetilde{\alpha}_{0}=(1,1,1,0) \notin M$. Assume that $\widetilde{\alpha}_{0}=(1,1,1,0) \in M$. Then there is another point $\widetilde{\alpha}_{1}=\left(a_{1,1}, a_{1,2}, a_{1,3}, 0\right) \notin M$ with $a_{1, i}=0$ for some $i=1,2,3$. Without loss of generality, applying a projective transformation permuting coordinates and preserving $P$ fixed, we can assume that this point is $\widetilde{\alpha}_{1}=\left(0, a_{1,2}, a_{1,3}, 0\right)$. Then $|M \cap l|=2$, where the line $l \subset \mathbb{P}^{3}$ is given by equations $a_{1}=a_{4}=0$. Therefore we have the only three points belonging to $M$ whose first coordinate $a_{1}$ vanishes, namely, two points lying in $M \cap l$ and the point $(0,0,0,1)$. Therefore $\left|M \cap P_{1}\right|=3$, where the plane $P_{1}=\left\{\widetilde{\alpha}=\left(a_{1}, a_{2}, a_{3}, a_{4}\right) \in \mathbb{P}^{3} \mid a_{1}=0\right\}$. Contradiction.

Let us show that the case $0<n_{i} \leqslant 4$ for all planes $P_{i} \subset \mathbb{P}^{3}$ is impossible. First of all, assume that $n_{i}=4$ for all planes $P_{i}$. On the one hand, the number of planes in $\mathbb{P}^{3}$ is equal to 15 . On the other hand, the number of planes passing through a point $\widetilde{\alpha} \in \mathbb{P}^{3}$ is equal to 7 and the inequality $4 \cdot 15 \neq 8 \cdot 7$ implies that this case is impossible.

Finally, assume that $0<n_{i} \leqslant 4$ for all planes $P_{i} \subset \mathbb{P}^{3}$ and there is a plane $P_{1}$ such that $n_{1}=\left|M \cap P_{1}\right|=2$. Then there exists a line $l \subset P_{1}$ such that $M \cap l=\emptyset$. Consider the pencil of planes passing through $l$. It consists of three planes, $P_{1}, P_{2}$, and $P_{3}$. Since $n_{1}+n_{2}+n_{3}=8$, we have $n_{2}+n_{3}=6$. Therefore, we can assume that $n_{2}=2$ and $n_{3}=4$. We have $M \cap P_{3}=P_{3} \backslash l$ and hence each plane $P$ not containing $l$ has two common points with $P_{3}$ belonging to $M$. On the other hand, let $\left\{\widetilde{\alpha}_{1}, \widetilde{\alpha}_{2}\right\}=M \cap P_{1}$ and $\left\{\widetilde{\alpha}_{3}, \widetilde{\alpha}_{4}\right\}=M \cap P_{2}$. Then the plane $P_{4}$ passing through the points $\widetilde{\alpha}_{1}, \widetilde{\alpha}_{2}$, and $\widetilde{\alpha}_{3}$ does not contain the line $l$. Therefore $n_{4} \geqslant 5$. Contradiction.

Let us return to the proof of Proposition [3, Let the lines of a line arrangement $\mathcal{L}$ consisting of eight lines be numerated by the points of a totally even set $M \subset$ $(\mathbb{Z} / 2 \mathbb{Z})^{4}, \mathcal{L}=\mathcal{L}_{M}=\bigcup_{\widetilde{\alpha}_{i} \in M} L_{\widetilde{\alpha}_{i}}$. The line arrangement $\mathcal{L}_{M}$ defines an epimorphism $\psi: H_{1}\left(\mathbb{P}^{2} \backslash \mathcal{L}_{M}, \mathbb{Z}\right) \rightarrow(\mathbb{Z} / 2 \mathbb{Z})^{4}$ given by $\psi\left(\lambda_{\widetilde{\alpha}_{i}}\right)=\widetilde{\alpha}_{i}$ and the epimorphism $\psi$ defines a $(\mathbb{Z} / 2 \mathbb{Z})^{4}$-Galois covering $h: Y \rightarrow \mathbb{P}^{2}$ branched along $\mathcal{L}_{M}$. Since the epimorphism $\psi$ is defined by $h$ only up to automorphism of $(\mathbb{Z} / 2 \mathbb{Z})^{4}$, therefore by Lemma 3 , we can assume that $M$ is either $M=\left\{\widetilde{\alpha}=\left(a_{1}, a_{2}, a_{3}, a_{4}\right) \in \mathbb{P}^{3} \mid a_{4}=1\right\}$ (type $I$ ) or

$$
\begin{aligned}
M=\{ & (1,0,0,0),(1,1,0,0),(0,1,0,0),(1,0,1,0),(0,0,1,0), \\
& (0,1,1,0),(1,1,1,1),(0,0,0,1)\}
\end{aligned}
$$

(type $I I$ ). Now, to complete the proof, by Remark 3, it suffices to note that the set of line arrangements $\mathcal{L}_{M}$, where $M$ is of type $I$ (respectively, of type $I I$ ), is an everywhere dense Zariski open (and, consequently, connected) subset of $\left(\mathbb{P}^{2}\right)^{8}$. 
Theorem 2. Let $X$ be a surface of general type, and let $f_{1}: Y_{1} \rightarrow X, f_{2}: Y_{2} \rightarrow X$ be two numerically $(d, m)$-canonical totally ramified cyclic coverings defined, respectively, by divisors $C_{1} \sim m K_{X}+\alpha_{1}$ and $C_{2} \sim m K_{X}+\alpha_{2}$, where $\alpha_{1}$ and $\alpha_{2}$ are numerically equivalent to zero and such that $\delta\left(\alpha_{1}\right)=\delta\left(\alpha_{2}\right)$. If $d m \geqslant 5$ then $Y_{1}$ and $Y_{2}$ are deformation equivalent.

Proof. Let $B_{i} \in\left|d C_{i}\right|$ be the branch curve of the covering $f_{i}, i=1,2$. The covering $f_{i}$ is given by adding a function $w_{i}$ to the field $\mathbb{C}(X)$ with $w_{i}^{d}=g_{i},\left(g_{i}\right)=B_{i}-d C_{i}$, $C_{i} \sim m K_{X}+\alpha_{i}$. Let $\Delta=\delta\left(\alpha_{1}\right)=\delta\left(\alpha_{2}\right) \in \operatorname{Tor}(X)$. Denote by $\operatorname{Pic}_{\Delta}(X)=\delta^{-1}(\Delta) \subset$ $\operatorname{Pic}(X)$.

Consider the scheme $T_{\Delta, d m}$ parametrizing the curves $B_{t}$ in $X$ numerically equivalent to $d m K_{X}$ and such that $\delta\left(B_{t}\right)=d m \delta\left(K_{X}\right)+d \Delta$ (the scheme $T_{\Delta, d m}$ is fibered over $\operatorname{Pic}_{\Delta}(X), \gamma_{1}: T_{\Delta, d m} \rightarrow \operatorname{Pic}_{\Delta}(X)$, with fibers $\gamma_{1}^{-1}\left(\alpha_{t}\right)=\mathbb{P}\left(H^{0}\left(X, \mathcal{O}_{X}\left(d m K_{X}+d \alpha_{t}\right)\right)\right.$ over $\alpha_{t} \in \operatorname{Pic}_{\Delta}(X)$; see [16]). Obviously, the subscheme $U$ consisting of points $t$ of $T_{\Delta, d m}$ for which $B_{t}$ are smooth curves is a Zariski open non-empty subset.

Similarly, let $T_{\Delta, 2}$ be the scheme parametrizing the curves $D_{t}$ in $X$ numerically equivalent to $2 K_{X}$ and such that $\delta\left(D_{t}\right)=2 \delta\left(K_{X}\right)+\Delta$. The scheme $T_{\Delta, 2}$ also is fibered over $\operatorname{Pic}_{\Delta}(X), \gamma_{2}: T_{\Delta, 2} \rightarrow \operatorname{Pic}_{\Delta}(X)$, with fibers $\gamma_{2}^{-1}\left(\alpha_{t}\right)=\mathbb{P}\left(H^{0}\left(X, \mathcal{O}_{X}\left(2 K_{X}+\alpha_{t}\right)\right)\right.$ over $\alpha_{t} \in \operatorname{Pic}_{\Delta}(X)$. Denote by $T=U \times_{\operatorname{Pic}_{\Delta}(X)} T_{\Delta, 2}$ the product of the fibrations $\gamma_{1}$ and $\gamma_{2}$ and let $p_{i}, i=1,2$, be the projections of $T$ onto the factors.

Let us fix a divisor $D \sim(m-2) K_{X}$ and associate with each $t \in T$ the divisor $C_{p_{2}(t)}=D_{p_{2}(t)}+D \sim m K_{X}+\alpha_{p_{2}(t)}$. By Lemma 2, the coverings $f_{1}$ and $f_{2}$ are defined by divisors $C_{p_{2}\left(t_{i}\right)}=D_{p_{2}\left(t_{i}\right)}+D \sim m K_{X}+\alpha_{p_{2}\left(t_{i}\right)}$ for some points $t_{i} \in T, i=1,2$, and branched along the curves $B_{p_{1}\left(t_{i}\right)}$. The family of divisors $D_{t}=B_{p_{1}(t)}-d C_{p_{2}(t)}$ defines a divisor $\widetilde{D}$ in $X \times T$ such that $\widetilde{D} \cap(X \times\{t\})=D_{t}$ for each $t \in T$. By Corollary 6 in [17, there is an invertible sheaf $\mathcal{L}$ on $T$ such that $\mathcal{O}_{X \times T}(\widetilde{D})=p^{*}(\mathcal{L})$, where $p: X \times T \rightarrow T$ is the projection. Obviously, we can choose a rational section of $\mathcal{L}$ the support of whose divisor $L$ does not contain the points $t_{1}$ and $t_{2}$. Let us consider a rational function $\widetilde{g}=g_{t}, t \in T, t \notin \operatorname{Supp} L$, such that the divisor $(\widetilde{g})$ is $\widetilde{D}-p^{*}(L)$. The function $\widetilde{g}$ defines a cyclic covering $\widetilde{f}: \widetilde{Y} \rightarrow X \times T$ given by $\widetilde{w}^{d}=\widetilde{g}$. This covering can be considered as a connected family of cyclic coverings $f_{t}: Y_{t} \rightarrow X$, $t \in T \backslash \operatorname{Supp} L$ given by $C_{p_{2}(t)}=D_{p_{2}(t)}+D \sim m K_{X}+\alpha_{p_{2}(t)}$ and branched along the curves $B_{p_{1}\left(t_{i}\right)}$, and hence $Y_{1}$ and $Y_{2}$ are deformation equivalent.

Remark 5. Theorem 2 is true without assumption $d m \geqslant 5$ if for each $\alpha \in \mathrm{Pic}_{\Delta}$ a generic curve $B \sim d\left(m K_{X}+\alpha\right)$ is smooth.

Theorem 3. Let $X$ be a surface of general type with $p_{g}=0$, and let $f: Y \rightarrow X$ be a numerically $(2, m)$-canonical totally ramified cyclic covering of $X$. Then the rational map $\varphi_{K_{Y}}: Y \rightarrow \mathbb{P}^{p_{g}(Y)-1}$ factorizes through $f$, that is, there exists a rational map $\psi: X \rightarrow \mathbb{P}^{p_{g}(Y)-1}$ such that $\varphi_{K_{Y}}=\psi \circ f$. 
Proof. Let $B \equiv 2 m K_{X}$ be the branch curve of $f$ and let $f$ be defined by a divisor $C \equiv m K_{X}$. As in the proof of Theorem 1, due to the projection formula for the canonical divisor we have the equality

$$
K_{Y}=f^{*}\left(K_{X}+C\right) .
$$

As any surface of general type with $p_{g}=0$, the surface $X$ is regular (that is, $q(X)=0$ ), so that Mumford Vanishing Theorem and Riemann-Roch Theorem imply

$$
\operatorname{dim} H^{0}\left(X, \mathcal{O}_{V}\left(K_{X}+C\right)\right)=\frac{\left(K_{X}+C, C\right)_{X}}{2}+1=\frac{m(m+1)}{2} K_{X}^{2}+1 .
$$

By Proposition 1, the surface $Y$ is also regular; therefore, applying the formula (3) from Proposition 2 we obtain

$$
p_{g}(Y)=1+\frac{m(m+1)}{2} K_{X}^{2}
$$

Now, the theorem follows from (5) - (7).

Corollary 1. Let $X$ be a surface of general type with $p_{g}=0$, and let $f: Y \rightarrow X$ be a numerically $(2, m)$-canonical totally ramified cyclic covering of $X$ defined by a divisor $C \equiv m K_{X}$. Then for $m \geqslant 4$ the canonical map $\varphi_{K_{Y}}: Y \rightarrow \mathbb{P}^{p_{g}(Y)-1}$ is a morphism of degree 2 over its image, and this image, $\varphi_{K_{Y}}(Y) \subset \mathbb{P}^{p_{g}(Y)-1}$, coincides with the image of $X$ under the birational morphism $\psi=\varphi_{K_{X}+C}$.

In the present time, there is a long list of known surfaces of general type with $p_{g}=0$ (see, for example, the survey [2]), but up to now, this list is far from completeness. In the following three propositions we investigate the degree of the canonical map of a pure $(2,1)$-canonical totally ramified cyclic covering, respectively, of a Campedelli surface, a Burniat surface, and a Mendes Lopes - Pardini surface.

Proposition 4. Let $f: Y \rightarrow X$ be a pure $(2,1)$-canonical totally ramified cyclic covering of a Campedelli surface $X$. Then $\varphi_{K_{Y}}: Y \rightarrow \mathbb{P}^{2}$ is a morphism of degree $\operatorname{deg} \varphi_{K_{Y}}=16$.

Proof. In notation used in the proof of Theorem 3, we have ([8]) $\varphi_{2 K_{X}}=\sigma \circ g$. Therefore, $\operatorname{deg} \varphi_{2 K_{X}}=8$. Now, Theorem 3 implies that $\varphi_{K_{Y}}=\varphi_{2 K_{X}} \circ f$, and thus the map $\varphi_{K_{Y}}$ is regular and of degree $\operatorname{deg} \varphi_{K_{Y}}=16$.

Proposition 5. Let $f: Y \rightarrow X$ be a pure $(2,1)$-canonical totally ramified cyclic covering of a Burniat surface $X$ with $K_{X}^{2} \geqslant 3$. Then $\varphi_{K_{Y}}: Y \rightarrow \mathbb{P}_{X}^{2}$ is a morphism of degree 8 over its image, and this image, $Z=\varphi_{K_{Y}}(Y)$, is a Del Pezzo surface with $K_{Z}^{2}=K_{X}^{2}$ embedded into $\mathbb{P}_{X}^{2}$ by means of the anticanonical map. 
Proof. Similar to Campedelli surfaces, a Burniat surface $X$ is the resolution of singularities of a Galois covering $\widetilde{g}: \widetilde{X} \rightarrow \mathbb{P}^{2}$ with Galois group $\operatorname{Gal}\left(\widetilde{X} / \mathbb{P}^{2}\right) \simeq(\mathbb{Z} / 2 \mathbb{Z})^{2}$ branched along a Burniat line arrangement $\widetilde{L}$ (see details in [5] or in [8]). To resolve the singularities, it suffices to blow up the $r$-fold points of the line arrangement $\widetilde{L}$ with $r \geqslant 3$ and consider the induced Galois covering $g: X \rightarrow \widetilde{\mathbb{P}}^{2}$, where $\sigma: \widetilde{\mathbb{P}}^{2} \rightarrow \mathbb{P}^{2}$ is the composition of blowups with centers at the $r$-fold points of $\widetilde{L}$, $r \geqslant 3$. The number of $r$-fold points of $\widetilde{L}$ with $r \geqslant 3$ is equal to $9-K_{X}^{2}$ and therefore $\widetilde{\mathbb{P}}^{2}$ is a Del Pezzo surface with $K_{\widetilde{\mathbb{P}}^{2}}^{2}=K_{X}^{2}$. Moreover, $2 K_{X}=g^{*}\left(-K_{\widetilde{\mathbb{P}}^{2}}\right)$ and $\operatorname{dim} H^{0}\left(X, \mathcal{O}_{X}\left(2 K_{X}\right)\right)=\operatorname{dim} H^{0}\left(\widetilde{\mathbb{P}}^{2}, \mathcal{O}_{\widetilde{\mathbb{P}} 2}\left(-K_{\widetilde{\mathbb{P}} 2}\right)\right)$ (see, for example, [8]). Therefore $\varphi_{2 K_{X}}=\varphi_{-K_{\tilde{\mathbb{P}} 2}} \circ \mathrm{g}$ is a regular map of degree four. Now, Theorem 3 shows that $\varphi_{K_{Y}}=\varphi_{2 K_{X}} \circ f$, which implies that $\operatorname{deg} \varphi_{K_{Y}}=8$.

Mendes Lopes and Pardini ([13]) constructed a six-dimensional family of surfaces $X$ of general type with $p_{g}=0$ and $K_{X}^{2}=3$. For each of these surfaces, call them Mendes Lopes - Pardini surfaces, the map $\varphi_{2 K_{X}}$ is regular of degree 2 over its image, $Z$, which is a singular Enriques surface $Z \subset \mathbb{P}^{3}, \operatorname{deg} Z=6$. We apply Theorem 3 following the same lines as above and obtain the following result.

Proposition 6. Let $f: Y \rightarrow X$ be a pure $(2,1)$-canonical totally ramified cyclic covering of a Mendes Lopes - Pardini surface $X$. Then $\varphi_{K_{Y}}: Y \rightarrow \mathbb{P}^{3}$ is a regular map of degree 4 over its image, $Z$, which is a singular Enriques surface $Z \subset \mathbb{P}^{3}$, $\operatorname{deg} Z=6$.

\section{CyClic COVERINGs of Rigid SURFACES}

Recall that each Miyaka-Yau surface $X$ being a holomorphic quotient of a ball satisfies Mostow rigidity theorem. The latter, in one of its well known formulations, states that (existence part) each element of the group Out $\pi_{1}(X)$ is realized by an antiholomorphic or holomorphic diffeomorphism $g: X \rightarrow X$, and furthermore (unicity part) such a realization is unique ( $c f .$, [15] and [7]).

Given a complex surface $X$, denote by $\bar{X}$ the surface with the complex conjugate complex structure and by $\mathrm{Kl}(X)$ the group of holomorphic and anti-holomorphic automorphisms of $X$.

Theorem 4. Let $X$ be a Miyaoka-Yau surface and let $f_{1}: Y_{1} \rightarrow X, f_{2}: Y_{2} \rightarrow X$ be two numerically $(d, m)$-canonical totally ramified cyclic coverings defined, respectively, by divisors $C_{1} \sim m K_{X}+\alpha_{1}$ and $C_{2} \sim m K_{X}+\alpha_{2}$, where both $\alpha_{1}, \alpha_{2}$ are numerically equivalent to zero. Assume, in addition, that in the case $d \geqslant 3$ both $\delta\left(\alpha_{1}\right)$ and $\delta\left(\alpha_{2}\right)$ have the same order $n$ in Tor $(X)$ coprime with $d-1$. If $Y_{1}$ and $Y_{2}$ are orientation preserving diffeomorphic, then there exists a (holomorphic or antiholomorphic) automorphism $\psi \in K l(X)$ such that $\psi^{*}\left(\delta\left(\alpha_{2}\right)\right)= \pm \delta\left(\alpha_{1}\right)$ (the sign is plus if $\psi$ is holomorphic, and minus otherwise). 
If $Y_{1}$ and $Y_{2}$ are deformation equivalent, then there exists an automoprhism $\psi \in$ Aut $(X)$ such that $\psi^{*}\left(\delta\left(\alpha_{2}\right)\right)=\delta\left(\alpha_{1}\right)$.

Proof. Let $\varphi: Y_{1} \rightarrow Y_{2}$ be an orientation preserving diffeomorphism.

We have

$$
\begin{aligned}
K_{Y_{1}}= & f_{1}^{*}\left(K_{X}\right)+(d-1) R_{1} \sim f_{1}^{*}\left(K_{X}\right)+(d-1) f_{1}^{*}\left(m K_{X}+\alpha_{1}\right) \sim \\
& (d m-d+1) f_{1}^{*}\left(K_{X}\right)+(d-1) f_{1}^{*}\left(\alpha_{1}\right)
\end{aligned}
$$

and, similarly, $K_{Y_{2}} \sim(d m-d+1) f_{2}^{*}\left(K_{X}\right)+(d-1) f_{2}^{*}\left(\alpha_{2}\right)$. The surfaces $Y_{1}$ and $Y_{2}$ being coverings of a surface of Kodaira dimension 2 have also Kodaira dimension 2 , and thus they are of general type. They are both minimal, since for any curve $E \subset Y_{i}$ we have $E \cdot K_{Y_{i}}=f_{*}(E) \cdot(d m-d+1) K_{X}>0$. Therefore, $\pm \delta\left(K_{Y_{i}}\right)$ are the only Seiberg-Witten basic classes in $H^{2}\left(Y_{i} ; \mathbb{Z}\right)$ (see [21] for the case $p_{g}>0$ and, for example, 4] for $\left.p_{g}=0\right)$, which implies $\varphi^{*}\left(\delta\left(K_{Y_{2}}\right)\right)= \pm \delta\left(K_{Y_{1}}\right)$.

By Proposition 10 the map $\varphi$ induces an automorphism $\varphi_{*}: \pi_{1}(X)=\pi_{1}\left(Y_{1}\right) \rightarrow$ $\pi_{1}\left(Y_{2}\right)=\pi_{1}(X)$ of $\pi_{1}(X)$ (more precisely, an element of Out $\pi_{1}(X)$ ), and therefore, in accordance with Mostow rigidity, it is induced by an either holomorphic or antiholomorphic automorphism $\psi: X \rightarrow X$. By Siu rigidity (see [20]), the map $f_{2} \circ \varphi: Y_{1} \rightarrow X$ is homotopic to either holomorphic or antiholomorphic morphism $\tilde{f}_{1}: Y_{1} \rightarrow X$ and, as it follows once more from Mostow rigidity, $\tilde{f}_{1}=\psi \circ f_{1}$ since these two morphisms define the same element of Out $\pi_{1}(X)$.

If $\psi$ is holomorphic then $\psi^{*}\left(K_{X}\right)=K_{X}$. Therefore $\varphi^{*}\left(f_{2}^{*}\left(\delta\left(K_{X}\right)\right)\right)=f_{1}^{*}\left(\delta\left(K_{X}\right)\right)$ and hence $\varphi^{*}\left((d-1) \delta\left(\alpha_{2}\right)\right)=(d-1) \delta\left(\alpha_{1}\right)$. Let us show that then $\varphi^{*}\left(\delta\left(\alpha_{2}\right)\right)=\delta\left(\alpha_{1}\right)$. Indeed, assume that $\varphi^{*}\left(\delta\left(\alpha_{2}\right)\right)=\delta\left(\alpha_{1}\right)+\beta$ for some $\beta \neq 0$ and such that $(d-1) \beta=0$. A priori, it is possible only if $d \geqslant 3$. But, in this case, since $(n, d-1)=1$, the element $\delta\left(\alpha_{1}\right)+\beta$ must have the order greater than $n$. On the other hand, the element $\varphi^{*}\left(\delta\left(\alpha_{2}\right)\right)$ has the same order as $\delta\left(\alpha_{2}\right)$. Therefore $\beta=0$ and $\psi^{*}\left(\delta\left(\alpha_{2}\right)\right)=\delta\left(\alpha_{1}\right)$.

If $\psi$ is antiholomorphic then $\psi^{*}\left(K_{X}\right)=-K_{X}$. Thus $\varphi^{*}\left(f_{2}^{*}\left(\delta\left(K_{X}\right)\right)\right)=-f_{1}^{*}\left(\delta\left(K_{X}\right)\right)$. Hence $\varphi^{*}\left((d-1) \delta\left(\alpha_{2}\right)\right)=-(d-1) \delta\left(\alpha_{1}\right)$ and as above we obtain that $\psi^{*}\left(\delta\left(\alpha_{2}\right)\right)=$ $-\delta\left(\alpha_{1}\right)$.

If $Y_{1}$ and $Y_{2}$ are deformation equivalent, then there is an orientation preserving diffeomorphism $\phi: Y_{1} \rightarrow Y_{2}$ such that $\varphi^{*}\left(\delta\left(K_{Y_{2}}\right)\right)=\delta\left(K_{Y_{1}}\right)$. Repeating once more the same arguments as above, we conclude that there exists $\psi \in \operatorname{Aut}(X)$ such that $\psi^{*}\left(\delta\left(\alpha_{2}\right)\right)=\delta\left(\alpha_{1}\right)$.

Corollary 2. Let $f_{1}: Y_{1} \rightarrow X$ and $f_{2}: Y_{2} \rightarrow X$ be numerically $(d, m)$-canonical totally ramified cyclic coverings as in Theorem 4. Suppose that $d m \geqslant 5$ and there exists an orientation preserving diffeomorphism $\varphi: Y_{1} \rightarrow Y_{2}$. Then $Y_{2}$ is deformation equivalent to $Y_{1}$ if $\varphi^{*}\left(K_{Y_{2}}\right)=K_{Y_{1}}$, and $\bar{Y}_{2}$ is deformation equivalent to $Y_{1}$ if $\varphi^{*}\left(K_{Y_{2}}\right)=-K_{Y_{1}}$. 
Proof. Let $B_{i} \in\left|d C_{i}\right|$ be the branch curve of the covering $f_{i}, i=1,2$. The covering $f_{i}$ is given by adding a function $w_{i}$ to the field $\mathbb{C}(X)$ with $w_{i}^{d}=g_{i},\left(g_{i}\right)=B_{i}-d C_{i}$, $C_{i} \sim m K_{X}+\alpha_{i}$.

According to Theorem 4, there exists $\psi \in \mathrm{Kl}(X)$ such that $\psi^{*}\left(\alpha_{2}\right)= \pm \alpha_{1}$ with sign "plus" if $\psi$ is holomorphic and sing "minus" otherwise. If $\psi$ is antiholomorphic, define an automorphism $\psi^{!}: \operatorname{Div}(X) \rightarrow \operatorname{Div}(X)$ by $\psi^{!}(D)=\psi^{-1}(D)$ in the case $D \subset X$ is a curve.

Let $\psi$ be a holomorphic automorphism. Then the covering $\psi^{-1} \circ f_{2}: Y_{2} \rightarrow X$ is given by adding a function $\widetilde{w}_{2}$ to the field $\mathbb{C}(X)$ with $\widetilde{w}_{2}^{d}=\psi^{*}\left(g_{2}\right),\left(\psi^{*}\left(g_{2}\right)\right)=$ $\psi^{*}\left(B_{2}\right)-d \psi^{*}\left(C_{2}\right)$, where $\psi^{*}\left(C_{2}\right) \sim m K_{X}+\psi^{*}\left(\alpha_{2}\right)$. By Theorem 2, $Y_{1}$ and $Y_{2}$ are deformation equivalent, since $\delta\left(\psi^{*}\left(\alpha_{2}\right)\right)=\delta\left(\alpha_{1}\right)$.

Let $\psi$ be an anti-holomorphic automorphism. Then the covering $\psi^{-1} \circ f_{2}: \bar{Y}_{2} \rightarrow X$ is given by adding a function $\bar{w}_{2}$ to the field $\mathbb{C}(X)$ with $\bar{w}_{2}^{d}=\psi^{*}\left(\bar{g}_{2}\right),\left(\psi^{!}\left(g_{2}\right)\right)=$ $\psi^{!}(B)-d \psi^{!}\left(C_{2}\right)$, where $\psi^{!}\left(C_{2}\right) \sim m K_{X}+\psi^{!}\left(\alpha_{2}\right)$. By Theorem 2, $Y_{1}$ and $\bar{Y}_{2}$ are deformation equivalent, since $\delta\left(\psi^{!}\left(\alpha_{2}\right)\right)=\delta\left(-\psi^{*}\left(\alpha_{2}\right)\right)=\delta\left(\alpha_{1}\right)$.

Corollary 3. Let $X$ be a Miyaoka-Yau surface and let $d \geq 2, m \geq 1$ be integers such that $d m \geq 5$ and $d-1$ is prime with respect to the order of the group Tor $(X)$. Then in the moduli space of surfaces the number of connected components that contain numerically $(d, m)$-canonical totally ramified cyclic coverings of $X$ is equal to the number of orbits of the action of $A u t(X)$ on $\operatorname{Tor}(X)$.

Proof. Let $f_{1}: Y_{1} \rightarrow X_{1}, f_{2}: Y_{2} \rightarrow X_{2}$ be two totally ramified numerically $(d, m)$ canonical cyclic coverings given by divisors $C_{1} \sim m K_{X}+\alpha_{1}, C_{2} \sim m K_{X}+\alpha_{2}$, respectively, where $\alpha_{1}$ and $\alpha_{2}$ are both numerically equivalent to zero. Assume that there exists an automorphism $\psi \in \operatorname{Aut}(X)$ such that $\psi^{*}\left(\delta\left(\alpha_{2}\right)\right)=\delta\left(\alpha_{1}\right)$. Then, $\psi^{-1} \circ f_{2}: Y_{2} \rightarrow X$ is a totally ramified numerically $(d, m)$-canonical cyclic covering given by divisor $\psi^{*}\left(C_{2}\right) \sim m K_{X}+\psi^{*}\left(\alpha_{2}\right)$ with $\delta\left(\psi^{*}\left(\alpha_{2}\right)\right)=\psi^{*}\left(\delta\left(\alpha_{2}\right)\right)=\delta\left(\alpha_{1}\right)$, and therefore, according to Theorem 2, the surfaces $Y_{1}$ and $Y_{2}$ belong to the same connected component of the moduli space.

The reverse statement follows from Theorem 4 ,

Theorem 5. Let $X$ be a Miyaoka-Yau surface and let $f_{1}: Y_{1} \rightarrow X, f_{2}: Y_{2} \rightarrow \bar{X}$ be two totally ramified numerically $(d, m)$-canonical cyclic coverings. If $Y_{1}$ and $Y_{2}$ are deformation equivalent, then $K l(X) \neq A$ ut $(X)$.

Proof. The covering $f_{2}$ provides also a totally ramified numerically $(d, m)$-canonical cyclic covering $f_{2}: \bar{Y}_{2} \rightarrow X$. As in the proof of Theorem 4 we have

$$
K_{Y_{1}} \equiv(d m-d+1) f_{1}^{*}\left(K_{X}\right) \quad \text { and } \quad K_{\bar{Y}_{2}} \equiv(d m-d+1) f_{2}^{*}\left(K_{X}\right) .
$$

Since $Y_{1}$ and $Y_{2}$ are deformation equivalent, there exists an orientation preserving diffeomorphism $\phi: Y_{1} \rightarrow Y_{2}$ such that $\phi^{*}\left(\delta\left(K_{\bar{Y}_{2}}\right)\right)=-\delta\left(K_{Y_{1}}\right)$. Therefore, we have

$$
\varphi^{*}\left(f_{2}^{*}\left(\delta\left(K_{X}\right)\right)\right) \equiv-f_{1}^{*}\left(\delta\left(K_{X}\right)\right) .
$$


The same arguments as in the proof of Theorem 4 shows that the group automorphism $\phi_{*}: \pi_{1}(X)=\pi_{1}\left(Y_{1}\right) \rightarrow \pi_{1}\left(Y_{2}\right)=\pi_{1}(X)$ (more precisely, the element of the group Out $\left.\pi_{1}(X)\right)$ is induced by a holomorphic, or anti-holomorphic, map $\psi: X \rightarrow X$ such that the map $f_{2} \circ \phi: Y_{1} \rightarrow X$ is homotopic to $\psi \circ f_{1}: Y_{1} \rightarrow X$, since the both maps define the same element of $\operatorname{Out} \pi_{1}(X)$.

Let us show that $\psi$ is an anti-holomorphic automorphism. Indeed, if $\psi$ is holomorphic, then $\psi^{*}\left(\delta\left(K_{X}\right)\right)=\delta\left(K_{X}\right)$. Therefore, it follows from equality (8) that

$$
-f_{1}^{*}\left(\delta\left(K_{X}\right)\right) \equiv \varphi^{*}\left(f_{2}^{*}\left(\delta\left(K_{X}\right)\right)\right)=f_{1}^{*}\left(\psi^{*}\left(\delta\left(K_{X}\right)\right)\right)=f_{1}^{*}\left(\delta\left(K_{X}\right)\right)
$$

but it is impossible since the element $f_{1}^{*}\left(\delta\left(K_{X}\right)\right)$ is not numerically equivalent to zero in $H^{2}\left(Y_{1}, \mathbb{Z}\right)$.

Corollary 4. For each pair of positive integers $d, m$ with $d m \geq 5, d \not \equiv 1(\bmod 5)$ and a surface $X$ of general type with $\left(K^{2}\right)_{X}=333$ and $e(X)=111$, the moduli space $\mathcal{M}_{k_{X, d, m}, p_{X, d, m}}$ has at least $3 \cdot 5^{6}$ different connected components.

Proof. Two surfaces $X$ as in the statement are constructed in Examples 1 and 2 in [9], denote them by $X_{1}$ and $X_{2}$ respectively. They are both obtained by resolution of singularities of the abelian $(\mathbb{Z} / 5 \mathbb{Z})^{2}$-coverings $\widetilde{h}_{i}: \widetilde{X}_{i} \rightarrow \mathbb{P}^{2}$ branched along the line arrangement $\widetilde{L}=\cup_{j=1}^{9} L_{j}$ dual to the inflection points of a smooth plane cubic. To resolve the singularities of $\widetilde{X}_{i}$, we blow up the 3 -fold points of $\widetilde{L}$ and take the normal closure of $\widetilde{\mathbb{P}}^{2}$ in the field $\mathbb{C}\left(\widetilde{X}_{i}\right)$; denote this blow up by $\sigma: \widetilde{\mathbb{P}}^{2} \rightarrow \mathbb{P}^{2}$ and the induced covering by $h_{i}: X_{i} \rightarrow \widetilde{\mathbb{P}}^{2}$.

The surfaces $\widetilde{X}_{i}$ can be obtained also as factor-spaces $Z / \widetilde{G}_{i}$, where $\widetilde{G}_{i} \simeq(\mathbb{Z} / 5 \mathbb{Z})^{6}$ and $Z$ is the abelian $(\mathbb{Z} / 5 \mathbb{Z})^{8}$-covering $g: Z \rightarrow \mathbb{P}^{2}$ defined by the field extension $g^{*}: \mathbb{C}\left(\mathbb{P}^{2}\right) \hookrightarrow \mathbb{C}(Z)=\mathbb{C}\left(\mathbb{P}^{2}\right)\left(w_{1}, \ldots, w_{8}\right)$ such that $w_{j}^{5}=l_{j} l_{9}^{-1}$, for $j=1, \ldots, 8$, and $l_{j}=0$ are equations of $L_{j}$. The divisors $\frac{1}{5} h_{i}^{*}\left(\left(\sigma^{*}\left(l_{j} l_{9}^{-1}\right)\right)\right)$ belong to $\operatorname{Tor}_{5}\left(X_{i}\right)$ and generate in it a subgroup $G_{i} \simeq(\mathbb{Z} / 5 \mathbb{Z})^{6}$.

From Proposition 2 it follows that the space $\mathcal{M}_{k_{X, d, m}, p_{X, d, m}}$ is non-empty, since by Bombieri Theorem the surface $X$ being of general type contains smooth curves numerically equivalent to $d m K_{X}$ if $d m \geqslant 5$.

By Proposition 4.1 in [9], $\operatorname{Kl}\left(X_{1}\right)=\operatorname{Aut}\left(X_{1}\right)=\operatorname{Gal}\left(\widetilde{X}_{1} / \mathbb{P}^{2}\right)$ and the action of $\operatorname{Aut}\left(X_{1}\right)$ on $G_{1}$ is trivial since $\operatorname{Kl}\left(X_{1}\right)$ leaves fixed the lines $L_{j} \subset \widetilde{L}$. Therefore, by Theorem 4, the surfaces $Y_{1, k}$ obtained as totally ramified numerically $(d, m)$-canonical coverings $f_{k}: Y_{1, k} \rightarrow X_{1}$ defined by the divisors $C_{k} \sim m K_{X}+\alpha_{k}, \alpha_{k} \in G_{1}$, are not pairwise orientation preserving diffeomorphic and hence they belong to distinct connected components of the moduli space $\mathcal{M}_{k_{X, d, m}, p_{X, d, m}}$.

According to Theorem 5, the surfaces $Y_{1}$ and $Y_{2}$ obtained as totally ramified numerically $(d, m)$-canonical cyclic coverings $f_{1}: Y_{1} \rightarrow X_{1}$ and $f_{2}: Y_{2} \rightarrow \bar{X}_{1}$ can not be deformation equivalent, since $\operatorname{Kl}\left(X_{1}\right)=\operatorname{Aut}\left(X_{1}\right)$. Therefore, the totally ramified numerically $(d, m)$-canonical cyclic coverings $f_{1, k}: Y_{k} \rightarrow \bar{X}_{1}$ defined by the divisors 
$C_{k} \sim m K_{\bar{X}_{1}}+\alpha_{k}, \alpha_{k} \in G_{1}$, define another $5^{6}$ distinct connected components of the moduli space $\mathcal{M}_{k_{X, d, m}, p_{X, d, m}}$.

Once more, by Proposition 4.1 in $[9], \operatorname{Aut}\left(X_{2}\right)=\operatorname{Gal}\left(\widetilde{X}_{2} / \mathbb{P}^{2}\right)$ and the action of $\operatorname{Aut}\left(X_{2}\right)$ on $G_{2}$ is trivial, while $\operatorname{Out}\left(\pi_{1}\left(X_{2}\right)\right)=\operatorname{Kl}\left(X_{2}\right) \neq \operatorname{Aut}\left(X_{2}\right)$. Hence, the totally ramified numerically $(d, m)$-canonical cyclic coverings $f: \tilde{Y} \rightarrow X_{2}$ give at least $5^{6}$ another distinct connected components in $\mathcal{M}_{k_{X, d, m}, p_{X, d, m}}$, since these surfaces $\tilde{Y}$ are not homeomorphic to the surfaces $Y$ obtained as totally ramified numerically $(d, m)$ canonical cyclic coverings $f: Y \rightarrow X_{1}$ (the fundamental groups of surfaces $\tilde{Y}$ and $Y$ have non isomorphic groups of outer automorphisms).

Theorem 6. Let $f_{1}: Y_{1} \rightarrow X$ be a totally ramified numerically $(2, m)$-canonical cyclic covering of a Miyaoka-Yau surface $X$ and let $\widetilde{Y}_{2}$ be a surface deformation equivalent to $Y_{1}$. Then the canonical model $Y_{2}$ of $\widetilde{Y}_{2}$ can be represented as a numerically $(2, m)$ canonical totally ramified cyclic covering of $X$ branched along a curve $B_{2} \subset X$, where $B_{2}$ is a reduced (not necessary irreducible) curve with ADE-singularities.

If $2 m \geqslant 5$, then the connected component $M$ of the moduli space to which $Y_{1}$ belongs is an irreducible variety of dimension $m(2 m-1) K_{X}^{2}+p_{g}(X)$. The Kodaira dimension $\kappa(M)$ of $M$ is equal to $-\infty$ and if the irregularity $q(X)=0$, then $M$ is an unirational variety.

Proof. Let $f_{1}: Y_{1} \rightarrow X$ be defined by a divisor $C \sim m K_{X}+\alpha_{1}$ and branched along a curve $B_{1} \in|2 C|$.

Since $Y_{1}$ and $\widetilde{Y}_{2}$ are deformation equivalent, there exists an orientation preserving diffeomorphism $\varphi: \widetilde{Y}_{2} \rightarrow Y_{1}$ such that $\varphi^{*}\left(K_{Y_{1}}\right)=K_{\widetilde{Y}_{2}}$. Hence, by Siu rigidity [20], the composition $f_{1} \circ \varphi$ is homotopic to a holomorphic map $h: \widetilde{Y}_{2} \rightarrow X$.

We have $\operatorname{deg} h=\operatorname{deg}\left(f_{1} \circ \varphi\right)=2$, and therefore $h^{*}: \mathbb{C}(X) \hookrightarrow \mathbb{C}\left(\widetilde{Y}_{2}\right)$ is a Galois extension of degree 2. Let $f_{2}: Y_{2} \rightarrow X$ be the normalization of $X$ in the field $\mathbb{C}\left(\widetilde{Y}_{2}\right)$ and let $\nu: \widetilde{Y}_{2} \rightarrow Y_{2}$ be a morphism such that $h=f_{2} \circ \nu$ (in fact, $\nu$ is the minimal resolution of singularities of $Y_{2}$ ).

Let us show that $Y_{2}$ has at most ADE-singularities. Indeed, if $D \subset \widetilde{Y}_{2}$ is an irreducible curve such that $\nu(D)$ is a point, then

$$
\left(K_{\widetilde{Y}_{2}}, D\right)_{\widetilde{Y}_{2}}=\left(\varphi^{*}\left(K_{Y_{1}}\right), D\right)_{\widetilde{Y}_{2}}=\left(\varphi^{*}\left(f_{1}^{*}\left(D_{1}\right)\right), D\right)_{\widetilde{Y}_{2}}=\left(h^{*}\left(D_{1}\right), D\right)_{\widetilde{Y}_{2}}=0,
$$

where $D_{1} \equiv m K_{X}+C \equiv(m+1) K_{X}$. It implies, by adjunction, that $D$ is a rational curve with $\left(D^{2}\right)_{\widetilde{Y}_{2}}=-2$.

Since $f_{2}: Y_{2} \rightarrow X$ is a double covering, its branch curve, which we denote by $B_{2}$, has similar to $Y_{2}$ at most ADE-singularities. Let us show that $B_{2} \equiv 2 m K_{X}$. Indeed, we have $2 K_{\widetilde{Y}_{2}}=h^{*}\left(2 K_{X}+B_{2}\right)$. On the other hand,

$$
2 K_{\widetilde{Y}_{2}}=2 \varphi^{*}\left(K_{Y_{1}}\right) \equiv 2(m+1) \varphi^{*}\left(f_{1}^{*}\left(K_{X}\right)\right) \equiv 2(m+1) h^{*}\left(K_{X}\right)
$$

and hence $B_{2} \equiv 2 m K_{X}$. 
By Remark 2, the covering $f_{2}: Y_{2} \rightarrow X$ is defined by a divisor $C_{2} \sim m K_{X}+\alpha_{2}$. A small deformation of $B_{2}$ into a smooth curve $B_{3} \sim B_{2}$ defines a numerically $(2, m)$ canonical (totally ramified) covering $f_{3}: Y_{3} \rightarrow X$ branched along $B_{3}$ and associated with the divisor $C_{2} \sim m K_{X}+\alpha_{2}$. Because of the existence of simultaneous resolution for simple singularities, $Y_{2}$ and $Y_{3}$ belong to the same irreducible component of the moduli space.

By Corollary 2 and Theorem 4, there is an automorphism $\psi \in \operatorname{Aut}(X)$ such that $\psi^{*}\left(\delta\left(\alpha_{2}\right)\right)=\delta\left(\alpha_{1}\right)$. Therefore, we can assume that $\delta\left(\alpha_{2}\right)=\delta\left(\alpha_{1}\right)$ (changing $f_{2}$ by $\left.\psi \circ f_{2}\right)$ and furthermore all the surfaces $Y_{t}$ deformation equivalent to $Y_{1}$ are numerically $(2, m)$-canonical totally ramified cyclic coverings of $X$ defined by $C_{t} \sim m K_{X}+\alpha_{t}$, where $\alpha_{t}$ is such that $\delta\left(\alpha_{t}\right)=\delta\left(\alpha_{1}\right)$, and branched along curves $B_{t} \sim 2\left(m K_{X}+\alpha_{t}\right)$ that have at most ADE-singularities.

Let $\Delta=\delta\left(\alpha_{1}\right) \in \operatorname{Tor}(X)$. Denote by $\operatorname{Pic}_{\Delta}(X)=\delta^{-1}(\Delta) \subset \operatorname{Pic}(X)$. As in the proof of Theorem 2 , consider the scheme $T_{\Delta, 2 m}$ parametrizing the curves $B_{t}$ in $X$ numerically equivalent to $2 m K_{X}$ and such that $\delta\left(B_{t}\right)=2 m \delta\left(K_{X}\right)+2 \Delta$. The scheme $T_{\Delta, 2 m}$ is fibered over $\operatorname{Pic}_{\Delta}(X), \gamma_{1}: T_{\Delta, 2 m} \rightarrow \operatorname{Pic}_{\Delta}(X)$, with fibers $\gamma_{1}^{-1}\left(\alpha_{t}\right)=\mathbb{P}\left(H^{0}\left(X, \mathcal{O}_{X}\left(2 m K_{X}+d \alpha_{t}\right)\right)\right.$ over $\alpha_{t} \in \operatorname{Pic}_{\Delta}(X)$. Obviously, the subscheme $U$ consisting of points $t$ of $T_{\Delta, 2 m}$ for which $B_{t}$ are reduced curves with at most ADE-singularities is a Zariski open non-empty subset.

Similarly, let $T_{\Delta, 2}$ be the scheme parametrizing the curves $D_{t}$ in $X$ numerically equivalent to $2 K_{X}$ and such that $\delta\left(D_{t}\right)=2 \delta\left(K_{X}\right)+\Delta$. The scheme $T_{\Delta, 2}$ also is fibered over $\operatorname{Pic}_{\Delta}(X), \gamma_{2}: T_{\Delta, 2} \rightarrow \operatorname{Pic}_{\Delta}(X)$, with fibers $\gamma_{2}^{-1}\left(\alpha_{t}\right)=\mathbb{P}\left(H^{0}\left(X, \mathcal{O}_{X}\left(2 K_{X}+\alpha_{t}\right)\right)\right.$ over $\widetilde{\alpha}_{t} \in \operatorname{Pic}_{\Delta}(X)$. Denote by $T=U \times_{\operatorname{Pic}_{\Delta}(X)} T_{\Delta, 2}$ the product of the fibrations $\gamma_{1}$ and $\gamma_{2}$ and let $p_{i}, i=1,2$, be the projections of $T$ onto the factors.

Note that $T$ is an irreducible variety and it follows from the above consideration and the proof of Theorem 2 that the points of $T$ parametrize all the surfaces deformation equivalent to $Y_{1}$. Therefore, the above considerations show that the connected component $M$ of the moduli space to which $Y_{1}$ belongs is an irreducible variety.

To complete the proof of Theorem, consider the surjective morphism $\mu: T \rightarrow M$. It is easy to see that the fibers of $p_{1}$ are subvarieties of the fibers of $\mu$. Let us show that each fiber of $\mu$ is the union of a finite number of fibers of $p_{1}$. Indeed, a degree two totally ramified covering $f: Y \rightarrow X$ branched along a curve $B \equiv 2 m K_{X}$ defines a degree two extension $\mathbb{C}(X) \hookrightarrow \mathbb{C}(Y)$. This extension defines an automorphism $f^{*} \in \operatorname{Aut}(Y)$ of order two whose set of fixed points is the ramification locus $R$ of $f$ (in the case when $Y$ has no $(-2)$-curves). Conversely, each $h \in \operatorname{Aut}(Y)$ determines uniquely the set of its fixed points. But, $\operatorname{Aut}(X)$ and $\operatorname{Aut}(Y)$ are finite groups, since $X$ and $Y$ are surfaces of general type. Therefore each fiber of $\mu$ is the union of a finite number of fibers of $p_{2}$ and, by Stein Factorization Theorem, the morphism $\mu$ factorizes through a finite morphism $\mu_{1}: U \rightarrow M_{1}$. 
We have $\operatorname{dim} M=\operatorname{dim} U=\operatorname{dim}\left|2 m K_{X}+d \alpha_{t}\right|+q(X)$. By Mumford Vanishing Theorem and Riemann-Roch Theorem,

$$
\operatorname{dim}\left|2\left(m K_{X}+\alpha_{1}\right)\right|=\frac{\left(2\left(m K_{X}+\alpha_{t}\right), 2\left(m K_{X}+\alpha_{t}\right)-K_{X}\right)_{X}}{2}+p_{g}(X)-q(X) .
$$

Therefore $\operatorname{dim} M=m(2 m-1) K_{X}^{2}+p_{g}(X)$. Moreover, since $U$ has Kodaira dimension $\kappa(U)=-\infty, M$ also has the same Kodaira dimension. If $q=0$, then $U$ is a rational variety and, therefore, $M$ is unirational.

Remark 6. The same arguments as in the proof of Theorem 6 show that for any $d \geqslant 2$ and for any surface $Y_{2}$ deformation equivalent to a surface $Y_{1}$ that is obtained as a numerically $(d, m)$-canonical totally ramified cyclic covering of a Miyaoka-Yau surface $X$, there exists a degree $d$ morphism $f_{2}: Y_{2} \rightarrow X$; but if $d \geqslant 3$, then $f_{2}$ is not necessary a cyclic covering. Furthermore, these arguments give rise to a lower bound on the dimension of that irreducible component $M$ of the moduli space of surfaces to which $Y_{1}$ belongs:

$$
\operatorname{dim} M \geqslant \frac{d m(d m-1)}{2} K_{X}^{2}+p_{g}(X)
$$

Remark 7. The proof of Theorem 6 shows that for any surface $Y$ that is a totally ramified numerically $(2, m)$-canonical cyclic covering of a Miayoka-Yau surface $X$, the action of the group $\mathbb{Z} / 2 \mathbb{Z}$ on $Y$ by deck transformation deforms simultaneously with any deformation of complex structure.

\section{NeW EXAmples of SURFACES HAVING NO ANY ANTi-HOLOMORPhiC AUTOMORPHISM}

In this section, by the Mostow strong rigidity of a compact complex manifold $X$ we mean the following property: whatever is a homotopy equivalence $p: X \rightarrow X$, it is homotopic to a holomorphic or anti-holomorphic map $X \rightarrow X$. By Mostow rigidity theorem, the fake projective planes and the surfaces from Examples 1 and 2 in [9] are Mostow strongly rigid. In addition, they are $K(\pi, 1)$ as topological spaces.

Let us underline that here we do not include the unicity statement into the definition of Mostow strong rigidity; the reason is that such a unicity statement is not used in the proofs of the results below. (Note, however, that if the definition had included the unicity, then in the statement given in Remark 9 the "if" could be replaced by the "if and only if".)

Theorem 7. Let $X$ be a Mostow strongly rigid surface of general type. If $X$ is a $K(\pi, 1)$ and $\operatorname{Out}(\pi)$ contains no elements of even order, then no numerically $(d, m)$ canonical totally ramified cyclic covering $Y$ of $X$ can be deformed to a surface isomorphic to $\bar{Y}$. In particular, all the surfaces $Y^{\prime}$ obtained by deformation of such a $Y$ have no any anti-holomorphic automorphism. 
Proof. We argue by contradiction. Assume that $Y$ is deformed to a surface $Y^{\prime}$ that is isomorphic to $\bar{Y}$. Identify $Y^{\prime}$ as a smooth manifold with $Y$ (in accordance with the deformation between them) and denote by $c: Y \rightarrow Y^{\prime}$ an anti-holomorphic diffeomorphism between $Y$ and $Y^{\prime}$. Preserve the same notation $c$ for the diffeomorphism induced by $c$ on $Y$ following our identification of the smooth manifolds underlying $Y$ and $Y^{\prime}$.

Consider the element $c_{*} \in \operatorname{Out}\left(\pi_{Y}\right)$ induced by $c$ (and the deformation equivalence between $Y$ and $Y^{\prime}$ ). By Proposition [1, $\pi_{1}(Y)=\pi_{1}(X)$. Since $X$ is Mostow strongly rigid, is of general type, and is $K(\pi, 1)$, the element $c_{*}$, as any element of $\operatorname{Out}\left(\pi_{X}\right)=$ $\operatorname{Out}\left(\pi_{Y}\right)$, is of finite order. Denote by $n$ the order of $c_{*}, n>0$. Since $X$ is a $K(\pi, 1)$, Proposition 1 implies that the maps $f: Y^{\prime}=Y \rightarrow X$ and $f \circ c^{n}$ are homotopic, and since in addition $c^{*}\left(\delta\left(K_{Y}\right)\right)=-\delta\left(K_{Y^{\prime}}\right)=-\delta\left(K_{Y}\right)$ (the Chern class $\delta(K)$ being integral does not change under deformations) while $f^{*}$ transforms $(d m-m+1) \delta\left(K_{X}\right)$ in $\delta\left(K_{Y}\right)$ modulo torsion, it implies that $n$ is even. Contradiction.

Remark 8. Literally the same proof shows that the surfaces $Y$ (and $Y^{\prime}$ ) satisfying Theorem 7 assumptions have no diffeomorphisms $f: Y \rightarrow Y$ with $f^{*}[K]=$ $-[K],[K] \in H^{2}(Y ; \mathbb{Q})$. Hence, due to the invariance of the canonical class under deformations in the class of almost-complex structures, the imaginary part $\omega$ of the Kähler structure of $Y$ considered as a symplectic structure on the underlying smooth manifold is not symplectic deformation equivalent to its reverse, $-\omega$. Thus, Theorem 7 and, in particular, its Corollary 5 below provide new examples of opposite symplectic structures not equivalent to each other (cf., [10]).

Remark 9. If $X$ is a Mostow strongly rigid surface of general type and $X$ is a $K(\pi, 1)$, then the fundamental group $\pi_{1}(X)$ has no automorphisms of even order $>0$ as soon as $X$ has no neither any anti-holomorphic automorphism or any holomorphic automorphism of non-zero even period.

Corollary 5. If $X$ is a fake projective plane or the rigid surface constructed in Example 1 in [9], then each of the surfaces $Y^{\prime}$ obtained by deformation of a numerically $(d, m)$-canonical totally ramified cyclic covering $Y$ of $X$ can not be deformed to its complex conjugate, and, in particular, has no any anti-holomorphic automorphism.

Proof. As is proved in [9], each of these surfaces $X$ has neither anti-holomorphic automorphisms nor holomorphic automorphisms of even order $>0$, so that the result follows from Theorem 7 and Remark 9.

\section{REFERENCES}

[1] Barth W., Hulek K., Peters C., Van de Ven A. Compact complex surfaces, Second edition, Springer-Verlag, Berlin, 2004.

[2] Bauer I., Catanese F, Pignatelli R.: Surfaces with geometric genus zero: asurvey. Ebeling, Wolfgang (ed.) et al. Complex and differntial geometry. Conference held at Leibniz Universitat Hannover, Germany, September $14-18$, 2009. Proceedings. Berlin: Springer. Springer Proceedings in Mathematics 8 (2011), 1 - 48. 
[3] Bombieri E.: Canonical models of surfaces of general type. Publ. Math. IHES, tome 42 (1973), $171-219$.

[4] Brussee R.: The Canonical Class and the $C^{\infty}$-properties of Kähler Surfaces. New York J. Math. 2 (1996), 103- 146.

[5] Burniat P.: Sur les surfaces de genre $P_{12}>0$. Ann. Pura Appl. (4) 71 (1966), $1-24$.

[6] Catanese F.: Moduli spaces of surfaces and real structures. Ann. Math. (2) 158 : 2 (2003), 577 $-592$.

[7] Gromov M., Pansu P.: Rigidity of Lattices : An Introduction. Geometric topology: recent developments, Lect. 1st Sess. CIME, Montecatini Terme/Italy 1990, Lect. Notes Math. 1504 (1991), $39-137$.

[8] Kulikov, Vik.S.: Old examples and a new example of surfaces of general type with $p_{g}=0$. Izv. Math. 68:5 (2004), $965-1008$.

[9] Kharlamov V., Kulikov Vik.S.: On real structures on rigid surfaces. Izv. Math., 66:1 (2002), $133-150$.

[10] Kharlamov V., Kulikov Vik.S.: Deformation inequivalent complex conjugated complex structures and applications. Turk. J. Math. 26 (2002), $1-25$.

[11] Kharlamov V., Kulikov Vik.S.: Surfaces with DIF $\neq$ DEF real structures. Izv. Math., 70 : 4 (2006), $769-807$.

[12] Manetti M.: On the moduli space of diffeomorphic algebraic surfaces. Invent. Math. 143 : 1 (2001), $29-76$.

[13] Mendes Lopes M., Pardini R.: A new family of surfaces with $p_{g}=0$ and $K^{2}=3$. Ann. Sci. Ec. Norm. Super. (4) 37 (2004), no. 4, $507-531$.

[14] Morgan J.W.: The Seiberg-Witten equations and application to the topology of smooth four manifolds. Math. Notes, 44, Princeton Univ Press vi (1996).

[15] Mostow G.D.: Strong rigidity of locally symmetric spaces. Annals of mathematics studies 78, Princeton University Press, Princeton, N.J. 1973.

[16] Mumford D.: Lectures on curves on an algebraic surface. With a section by G. M. Bergman. Annals of Mathematics Studies, No. 59 Princeton University Press, Princeton, N.J. 1966.

[17] Mumford D.: Abelian varieties. Lectures in Tata Institute of Fundamental Research, Bombay, 1968.

[18] Nori M.V.: Zariski's conjecture and related problems. Ann.Sci. Ecole Norm. Sup. (4) 16 (1983), no. 2, $305-344$.

[19] Reider I.: Vector bundles of rank 2 and linear systems on algebraic surfaces. Ann. of Math. (2) $127: 2$ (1988), 309-316.

[20] Siu Y.-T.: The Complex-Analyticity of Harmonic Maps and the Strong Rigidity of Compact Kahler Manifolds. Annals of Mathematics Second Series, 112 :1 (1980), 73 - 111.

[21] Witten E.: Monopoles and Four-Manifolds. Math. Res. Lett. 1 (1994), 809 - 822.

Strasbourg UNIVERSITY

E-mail address: kharlam@math.unistra.fr

Steklov Mathematical Institute

E-mail address: kulikov@mi.ras.ru 